\title{
A metric on the space of projections admitting nice isometries
}

by

Lajos Molnár (Debrecen) and Werner Timmermann (Dresden)

Dedicated to Professor Zoltán Daróczy on the occasion of his seventieth birthday

\begin{abstract}
Motivated by the concept of separation between propositions in quantum logic, we introduce the so-called separation metric or Santos metric on the space of all projections in a Hilbert space. We show that the resulting metric space has only "nice" surjective isometries. On the nontrivial projections they are all unitarily or antiunitarily equivalent to the identity or to taking the orthogonal complement. We relate this result to Wigner's classical theorem on the form of quantum mechanical symmetry transformations.
\end{abstract}

1. Introduction and statement of the main result. In [9] Santos defined the separation $S_{\mu}(p, q)$ between quantum mechanical propositions $p, q$ in a given state $\mu$ as

$$
S_{\mu}(p, q)=\mu(p)+\mu(q)-2 \mu(p \wedge q) .
$$

He proved that if the underlying lattice of propositions is a Boolean orthocomplemented lattice, then $S_{\mu}$ is a pseudometric. Furthermore, he showed that the famous Bell inequalities which play a fundamental role in the foundations of quantum mechanics are easily derivable from the triangle inequality for $S_{\mu}$. In this way he could present a new approach to the Bell inequalities that stresses their fundamental and general character (see also [8]).

In the usual Hilbert space description of quantum mechanics, the propositions are represented by projections on a (complex) Hilbert space $H$ and the (pure) states by unit vectors in $H$. Let $\mathcal{P}(H)$ denote the lattice of all projections (i.e., self-adjoint idempotents) on $H$. For any $P, Q \in \mathcal{P}(H)$ and

2000 Mathematics Subject Classification: Primary 47B49; Secondary 54E40.

Key words and phrases: Hilbert space, projections, Santos metric, gap metric, surjective isometries. 
unit vector $x \in H$, the corresponding separation in the sense of Santos is

$$
S_{x}(P, Q)=\langle P x, x\rangle+\langle Q x, x\rangle-2\langle(P \wedge Q) x, x\rangle .
$$

Taking the supremum over all unit vectors and referring to the fact that the norm of a self-adjoint operator equals its numerical radius, we arrive at the quantity

$$
d_{\mathrm{S}}(P, Q)=\|P+Q-2 P \wedge Q\| \quad(P, Q \in \mathcal{P}(H)) .
$$

It turns out to be a metric on $\mathcal{P}(H)$. To see this, first observe that $P+Q-$ $2 P \wedge Q$ can be written as the sum of the projections $P_{0}=P-P \wedge Q$ and $Q_{0}=Q-P \wedge Q$. It then follows that if $P$ differs from $Q$ and hence one of the projections $P_{0}$ and $Q_{0}$ is nonzero, then $1 \leq d(P, Q) \leq 2$. Clearly, this property alone implies the validity of the triangle inequality. Suppose now that $d_{\mathrm{S}}(P, Q)=0$. Then $0=\|(P-P \wedge Q)+(Q-P \wedge Q)\|$. But if the sum of two projections is zero, then the projections themselves are necessarily zero. Therefore, $P=P \wedge Q=Q$ and, as the symmetry of $d_{\mathrm{S}}$ is obvious, it follows that $d_{\mathrm{S}}$ is really a metric. In what follows we call $d_{\mathrm{S}}$ the separation metric or Santos metric on $\mathcal{P}(H)$.

This metric has a certain geometrical meaning. To see this, suppose that $P, Q \in \mathcal{P}(H)$ are not comparable, i.e., neither $P \leq Q$ nor $Q \leq P$ (in the opposite case we have $d_{\mathrm{S}}(P, Q)=0$ or 1 depending on whether $P$ equals $Q$ or not). Set, as before, $P_{0}=P-P \wedge Q$ and $Q_{0}=P-P \wedge Q$. We then have

$$
d_{\mathrm{S}}(P, Q)=\left\|P_{0}+Q_{0}\right\| .
$$

The norm of the sum of two projections can be computed by using Vidav's theorem [13] saying that for any projections $e, f$ in a $C^{*}$-algebra we have $\|e+f\|=1+\|e f\|$ provided that $e, f$ are not both zero. Observing that $P_{0}, Q_{0} \neq 0$, we thus obtain $d_{\mathrm{S}}(P, Q)=1+\left\|P_{0} Q_{0}\right\|$. Set

$$
M_{0}=\operatorname{rng} P_{0}, \quad N_{0}=\operatorname{rng} Q_{0}
$$

(rng stands for the range of operators). Lemma 4.3 in [2] tells us that $\left\|P_{0} Q_{0}\right\|=\cos \left(M_{0}, N_{0}\right)$ where the cosine between nonzero subspaces is defined by

$$
\cos \left(M_{0}, N_{0}\right)=\sup \left\{\frac{|\langle m, n\rangle|}{\|m\|\|n\|}: 0 \neq m \in M_{0}, 0 \neq n \in N_{0}\right\} .
$$

Therefore,

$$
d_{\mathrm{S}}(P, Q)=1+\cos \left(M_{0}, N_{0}\right) .
$$

The above cosine between subspaces reminds us an important concept in quantum mechanics called transition probability, whose geometrical meaning is the (squared) cosine of the angle between one-dimensional subspaces of a Hilbert space representing pure states. The bijective transformations on pure states preserving the transition probability are called quantum mechanical symmetry transformations. They are described by Wigner's famous theorem, 
which is of fundamental importance in the probabilistic aspects of quantum mechanics. It says that every such transformation is induced by either a unitary or an antiunitary operator on the underlying Hilbert space.

In recent years we have obtained several generalizations and extensions of Wigner's theorem. Here we refer only to the one that describes the structure of those maps which preserve the system of canonical angles on the set of all subspaces of a Hilbert space with some fixed dimension. The result appeared in [4] (see also [6], and Chapter 2 in [5] for further information and results).

By what we have just learnt about the Santos metric, it is clear that in the purely mathematical sense, the corresponding isometries of the projection lattice $\mathcal{P}(H)$ can be viewed as generalizations of quantum mechanical symmetry transformations from the case of rank-one projections to the entire set $\mathcal{P}(H)$. This gives the motivation to determine the isometries of this metric space, which is the main aim of the paper. We shall prove that every surjective isometry is of the form either $P \mapsto U P U^{*}$ or $P \mapsto U P^{\perp} U^{*}$ on the set of all nontrivial projections, where $U$ is a unitary or antiunitary operator.

Before turning to the details let us make some remarks concerning another operator-norm-related metric on $\mathcal{P}(H)$, called the gap metric:

$$
d_{\mathrm{g}}(P, Q)=\|P-Q\| \quad(P, Q \in \mathcal{P}(H)) .
$$

In terms of subspaces, this quantity has been originally defined by B. Szốkefalvi-Nagy and independently by M. G. Krein and M. A. Krasnosel'skiı̌ under the name "aperture" (see [1, Section 34]). The gap metric has important applications, for example, in control theory.

To relate $d_{\mathrm{g}}$ to the Santos metric $d_{\mathrm{S}}$, observe that for any $P, Q \in \mathcal{P}(H)$, setting $P_{0}=P-P \wedge Q$ and $Q_{0}=Q-P \wedge Q$ as above, we have

$$
d_{\mathrm{g}}(P, Q)=\|P-Q\|=\left\|P_{0}-Q_{0}\right\|,
$$

while we recall that

$$
d_{\mathrm{S}}(P, Q)=\left\|P_{0}+Q_{0}\right\| .
$$

Moreover, by the so-called Akhiezer-Glazman equality (see [1, Section 34]),

$$
\begin{aligned}
d_{\mathrm{g}}(P, Q) & =\left\|P_{0}-Q_{0}\right\|=\max \left\{\left\|P_{0} Q_{0}^{\perp}\right\|,\left\|P_{0}^{\perp} Q_{0}\right\|\right\} \\
& =\max \left\{\cos \left(M_{0}, N_{0}^{\perp}\right), \cos \left(M_{0}^{\perp}, N_{0}\right)\right\} .
\end{aligned}
$$

This shows that both $d_{\mathrm{S}}$ and $d_{\mathrm{g}}$ are intimately related to angles between the ranges of $P$ and $Q$ after eliminating their intersection. However, there are serious differences, for example, in the structure of the corresponding isometry groups. In fact, one can immediately observe that $\mathcal{P}(H)$ equipped with the gap metric has a quite complicated isometry group containing in some sense rather "irregular" elements (which are not so nice as the ones for the 
Santos metric mentioned above). The reason for this is the following. For any $P, Q \in \mathcal{P}(H)$ we have $-I \leq P-Q \leq I$, implying that $d_{\mathrm{g}}(P, Q) \leq 1$. Moreover, it is well known that if $\|P-Q\|<1$, then $P, Q$ are unitarily equivalent (their ranges and kernels have the same Hilbert dimensions; see [1, Section $34]$ ). Now consider the corresponding equivalence classes of $\mathcal{P}(H)$. It is clear that if we have surjective isometries on each of those classes (which may be completely unrelated to each other, e.g., implemented by different unitary or antiunitary operators on different equivalence classes), then piecing them together to a map on the whole space $\mathcal{P}(H)$ we obtain a surjective isometry of $\mathcal{P}(H)$ which is "irregular" in the above sense.

We now turn to the result of this paper. Our aim is to determine the structure of all isometries of the space $\mathcal{P}(H)$ with respect to the Santos metric $d_{\mathrm{S}}$. Let us first give some particular examples of such isometries. It is apparent that for any unitary or antiunitary operator $U$ on $H$, the transformation $P \mapsto U P U^{*}$ is a surjective isometry. It is also rather obvious that any map $\pi$ on $\mathcal{P}(H)$ which permutes the set $\{0, I\}$ of trivial projections and acts as the identity on the remaining part of $\mathcal{P}(H)$ is also a surjective isometry.

To present a third example, we show the nontrivial fact that taking the orthogonal complement is also an isometry.

Proposition. The map $P \mapsto P^{\perp}=I-P$ is a surjective isometry of $\mathcal{P}(H)$ with respect to the metric $d_{\mathrm{S}}$.

So we have three different kinds of transformations which are all surjective isometries with respect to the Santos metric. Our main result states that in fact every surjective isometry can be obtained as a composition of transformations of these particular kinds.

Theorem. Let $H$ be a complex Hilbert space with $\operatorname{dim} H \geq 2$. Let $\phi$ : $\mathcal{P}(H) \rightarrow \mathcal{P}(H)$ be a surjective isometry with respect to the metric $d_{\mathrm{S}}$. Then $\phi$ permutes the set $\{0, I\}$ of trivial projections and there exists a unitary or antiunitary operator $U$ on $H$ such that either

$$
\phi(P)=U P U^{*} \quad(0, I \neq P \in \mathcal{P}(H)),
$$

or

$$
\phi(P)=U P^{\perp} U^{*} \quad(0, I \neq P \in \mathcal{P}(H)) .
$$

As already mentioned above, this result can be considered an extension of Wigner's theorem from the case of rank-one projections to the whole space of projections.

\section{Proofs}

Proof of the Proposition. Clearly, it is sufficient to verify that $d_{\mathrm{S}}(P, Q) \leq$ $d_{\mathrm{S}}\left(P^{\perp}, Q^{\perp}\right)$ for any $P, Q \in \mathcal{P}(H)$. The cases $P \leq Q$ and $Q \leq P$ are trivial, 
so suppose that $P$ and $Q$ are not comparable. Set

$$
M=\operatorname{rng} P, \quad N=\operatorname{rng} Q .
$$

Decompose the Hilbert space $H$ as the orthogonal sum of the closed subspaces $M \wedge N,(M \vee N) \wedge(M \wedge N)^{\perp}$ and $(M \vee N)^{\perp}$. Observe that $(M \vee N)$ $\wedge(M \wedge N)^{\perp} \neq\{0\}$. We have the corresponding matrix representations

$$
P=\left(\begin{array}{ccc}
I & 0 & 0 \\
0 & P_{0} & 0 \\
0 & 0 & 0
\end{array}\right), \quad Q=\left(\begin{array}{ccc}
I & 0 & 0 \\
0 & Q_{0} & 0 \\
0 & 0 & 0
\end{array}\right)
$$

Here $P_{0}$ and $Q_{0}$ are the restrictions of $P$ and $Q$ onto the subspace $(M \vee N) \wedge$ $(M \wedge N)^{\perp}$. We have

$$
\operatorname{rng} P_{0}=M \wedge(M \wedge N)^{\perp}, \quad \operatorname{rng} Q_{0}=N \wedge(M \wedge N)^{\perp},
$$

hence $P_{0} \wedge Q_{0}=0$. On the other hand, we assert that $P_{0} \vee Q_{0}$ equals the identity (on the space $\left.(M \vee N) \wedge(M \wedge N)^{\perp}\right)$. To see this, first note that

$$
M=\left(M \wedge(M \wedge N)^{\perp}\right) \vee(M \wedge N), \quad N=\left(N \wedge(M \wedge N)^{\perp}\right) \vee(M \wedge N) .
$$

It follows that

$$
M \vee N=\left(M \wedge(M \wedge N)^{\perp}\right) \vee\left(N \wedge(M \wedge N)^{\perp}\right) \vee(M \wedge N) .
$$

Since the last subspace on the right hand side is orthogonal to the previous ones, we obtain

$$
\left(M \wedge(M \wedge N)^{\perp}\right) \vee\left(N \wedge(M \wedge N)^{\perp}\right)=(M \vee N) \wedge(M \wedge N)^{\perp},
$$

and this proves $P_{0} \vee Q_{0}=I$.

Observe that $P_{0}, Q_{0}$ are nontrivial projections. Using the above representation, it is easy to see that

$$
d_{\mathrm{S}}(P, Q)=\left\|P_{0}+Q_{0}\right\|, \quad d_{\mathrm{S}}\left(P^{\perp}, Q^{\perp}\right)=\left\|P_{0}^{\perp}+Q_{0}^{\perp}\right\| .
$$

Applying Vidav's already mentioned result of [13], we obtain

$$
d_{\mathrm{S}}(P, Q)=1+\left\|P_{0} Q_{0}\right\|, \quad d_{\mathrm{S}}\left(P^{\perp}, Q^{\perp}\right)=1+\left\|P_{0}^{\perp} Q_{0}^{\perp}\right\| .
$$

We now distinguish two possibilities. First suppose that $0 \notin \sigma\left(P_{0}+Q_{0}\right)$. Then $P_{0}+Q_{0}$ is surjective and $\operatorname{rng} P_{0}$, rng $Q_{0}$ give a direct sum decomposition of the Hilbert space where these operators act. Next we apply a nice observation from [3]: if a Hilbert space is the direct sum of nontrivial closed subspaces $R$ and $K$, then the norm of the idempotent with range $R$ and kernel $K$ equals $\sqrt{1-\left\|P_{K} P_{R}\right\|^{2}}-1$ (here $P_{K}$ and $P_{R}$ denote the orthogonal projections onto $K$ and $R$, respectively). As mentioned in the proof of Theorem 2 in [3], it then follows immediately that $\left\|P_{K} P_{R}\right\|=\left\|P_{K}^{\perp} P_{R}^{\perp}\right\|$. Therefore, $\left\|P_{0} Q_{0}\right\|=\left\|P_{0}^{\perp} Q_{0}^{\perp}\right\|$, yielding $d_{\mathrm{S}}(P, Q)=d_{\mathrm{S}}\left(P^{\perp}, Q^{\perp}\right)$. 
Suppose now that $0 \in \sigma\left(P_{0}+Q_{0}\right)$. This implies that $2 \in \sigma\left(P_{0}^{\perp}+Q_{0}^{\perp}\right)$ and

$$
\left\|P_{0}+Q_{0}\right\| \leq 2=\left\|P_{0}^{\perp}+Q_{0}^{\perp}\right\|,
$$

which shows $d_{\mathrm{S}}(P, Q) \leq d_{\mathrm{S}}\left(P^{\perp}, Q^{\perp}\right)$.

Proof of the Theorem. First we assert that the commutativity of projections can be expressed in terms of the metric $d_{\mathrm{S}}$ as follows. For any $P, Q \in \mathcal{P}(H), P \neq Q$, we have

$$
P Q=Q P \Leftrightarrow d_{\mathrm{S}}(P, Q)=1 .
$$

To see this, we use the matrix representation from the proof of the Proposition. Observe that if $P, Q$ are comparable, then $P Q=Q P$ and $d_{\mathrm{S}}(P, Q)=1$ both hold. So, suppose that $P, Q$ are not comparable. Then $d_{\mathrm{S}}(P, Q)=$ $\left\|P_{0}+Q_{0}\right\|=1+\left\|P_{0} Q_{0}\right\|$. Consequently, $d_{\mathrm{S}}(P, Q)=1$ if and only if $P_{0} Q_{0}=0$. The latter equality implies that $P_{0}, Q_{0}$ commute, and hence $P, Q$ also commute. Conversely, if $P, Q$ commute, then so do $P_{0}, Q_{0}$ and we have $0=$ $P_{0} \wedge Q_{0}=P_{0} Q_{0}$, implying $d_{\mathrm{S}}(P, Q)=1$. This proves the asserted characterization of commutativity in $\mathcal{P}(H)$.

As $\phi$ is a surjective isometry, it follows that $\phi$ preserves commutativity in both directions. This means that for any $P, Q \in \mathcal{P}(H)$ we have $P Q=Q P$ if and only if $\phi(P) \phi(Q)=\phi(Q) \phi(P)$. At this point we can apply an argument similar to one in [7].

For any set $\mathcal{S} \subset \mathcal{P}(H)$ define

$$
\mathcal{S}^{\mathrm{k}}=\{Q \in \mathcal{P}(H): Q P=P Q(P \in \mathcal{S})\},
$$

which is the commutant of $\mathcal{S}$ relative to $\mathcal{P}(H)$. Moreover, denote by $\mathcal{S}^{\prime}$ the usual commutant of $\mathcal{S}$ in the algebra of all bounded linear operators on $H$. Using some elementary von Neumann algebra techniques, it is fairly easy to verify that $\mathcal{S}^{\mathrm{kk}}=\mathcal{S}^{\prime \prime} \cap \mathcal{P}(H)$.

This observation implies the following. If a finite sequence $P_{1}, \ldots, P_{n}$ of mutually orthogonal nonzero projections forms a resolution of the identity (i.e., its sum equals $I$ ), then $\left\{P_{1}, \ldots, P_{n}\right\}^{\mathrm{kk}}$ is precisely the set of all linear combinations of $P_{1}, \ldots, P_{n}$ which are projections, that is, the set of all linear combinations of $P_{1}, \ldots, P_{n}$ with coefficients 0 or 1 . Consequently, the set $\left\{P_{1}, \ldots, P_{n}\right\}^{\mathrm{kk}}$ has exactly $2^{n}$ elements.

Pick commuting projections $P, Q \in \mathcal{P}(H)$ and consider the corresponding resolution of the identity: $P \wedge Q, P-(P \wedge Q), Q-(P \wedge Q),(P \vee Q)^{\perp}$. We clearly have

$$
\{P, Q\}^{\mathrm{k}}=\left\{P \wedge Q, P-(P \wedge Q), Q-(P \wedge Q),(P \vee Q)^{\perp}\right\}^{\mathrm{k}}
$$

and hence

$$
\{P, Q\}^{\mathrm{kk}}=\left\{P \wedge Q, P-(P \wedge Q), Q-(P \wedge Q),(P \vee Q)^{\perp}\right\}^{\mathrm{kk}} .
$$


For temporary use, let us call a projection $P \in \mathcal{P}(H)$ simple if $P$ or $P^{\perp}$ is of rank one. One can easily check that a nontrivial projection $P$ is simple if and only if the set $\left\{P \wedge Q, P-(P \wedge Q), Q-(P \wedge Q),(P \vee Q)^{\perp}\right\}$ has at most three nonzero elements for every $Q \in \mathcal{P}(H)$ commuting with $P$. As a consequence, we have the following characterization of simple projections: the nontrivial element $P \in \mathcal{P}(H)$ is a simple projection if and only if the cardinality of the set $\{P, Q\}^{\mathrm{kk}}$ (which is already known to equal $2^{k}$, where $k$ is the number of nonzero elements of $\left.\left\{P \wedge Q, P-(P \wedge Q), Q-(P \wedge Q),(P \vee Q)^{\perp}\right\}\right)$ is at most 8 for every $Q \in \mathcal{P}(H)$ commuting with $P$.

As $\phi$ is a bijective map on $\mathcal{P}(H)$ which preserves commutativity in both directions, it follows that $\phi$ sends trivial projections to trivial projections and has the property that $\phi\left(\mathcal{S}^{\mathrm{kk}}\right)=\phi(\mathcal{S})^{\mathrm{kk}}$ for any $\mathcal{S} \subset \mathcal{P}(H)$. Therefore, by the above characterization of simple projections we see that $\phi$ preserves those elements in both directions, i.e., for any $P \in \mathcal{P}(H)$ we have: $P$ is simple if and only if $\phi(P)$ is simple.

In what follows let us suppose that $\operatorname{dim} H \geq 3$.

Define a new transformation $\psi$ on the set $\mathcal{P}_{1}(H)$ of all rank-one projections in the following way. For $P \in \mathcal{P}_{1}(H)$, let $\psi(P)=\phi(P)$ if $\phi(P)$ is of rank one, and let $\psi(P)=\phi(P)^{\perp}$ if $\phi(P)^{\perp}$ is of rank one. Clearly, $\psi$ is a well-defined map from $\mathcal{P}_{1}(H)$ into itself which preserves commutativity in both directions. To verify that $\psi$ is bijective we remark the following. Pick different commuting simple projections $P$ and $Q$. It is easy to see that $P+Q=I$ if and only if $\{P\}^{\mathrm{k}}=\{Q\}^{\mathrm{k}}$. The already known properties of $\phi$ imply that $\phi(P)+\phi\left(P^{\perp}\right)=I$ for every rank-one projection $P$. Now, to prove the injectivity of $\psi$ take rank-one projections $P$ and $Q$ such that $\psi(P)=\psi(Q)$. The nontrivial case that we have to consider is when one of $\phi(P)$ and $\phi(Q)$, say the first, is of rank one and the other is of corank one. But $\phi(P)=\phi(Q)^{\perp}=\phi\left(Q^{\perp}\right)$ implies $P=Q^{\perp}$, which is untenable. This proves the injectivity of $\psi$. The surjectivity can be verified in a similar way.

Therefore, we have a bijective map $\psi: \mathcal{P}_{1}(H) \rightarrow \mathcal{P}_{1}(H)$ which preserves commutativity in both directions. Since the commutativity of two different rank-one projections is equivalent to their orthogonality, it follows that $\psi$ preserves orthogonality in both directions. Such transformations are completely described by a famous theorem of Uhlhorn [12] which is a generalization of Wigner's theorem. According to that result, there is a unitary or antiunitary operator $U$ on $H$ such that $\psi$ is of the form

$$
\psi(P)=U P U^{*} \quad\left(P \in \mathcal{P}_{1}(H)\right) .
$$

Consider now the new transformation $\phi^{\prime}(P)=U^{*} \phi(P) U(P \in \mathcal{P}(H))$. This is obviously a surjective isometry of $\mathcal{P}(H)$ such that for every rank-one projection $P$ we have either $\phi^{\prime}(P)=P$ or $\phi^{\prime}(P)=P^{\perp}$. We show that either $\phi^{\prime}(P)=P$ for every $P \in \mathcal{P}_{1}(H)$, or $\phi^{\prime}(P)=P^{\perp}$ for every $P \in \mathcal{P}_{1}(H)$. 
To verify this, pick two unit vectors $x$ and $y$ in $H$ such that their inner product satisfies $|\langle x, y\rangle| \neq 0,1 / \sqrt{2}, 1$. Let $P_{x}$ and $P_{y}$ denote the rank-one projections onto the subspaces generated by $x$ and $y$, respectively. Suppose that $\phi^{\prime}\left(P_{x}\right)=P_{x}$ while $\phi^{\prime}\left(P_{y}\right)=P_{y}^{\perp}$. By Vidav's result [13] we have

$$
d_{\mathrm{S}}\left(P_{x}, P_{y}\right)=\left\|P_{x}+P_{y}\right\|=1+\left\|P_{x} P_{y}\right\|=1+\left\|P_{y} x\right\|
$$

and

$$
d_{\mathrm{S}}\left(P_{x}, P_{y}^{\perp}\right)=\left\|P_{x}+P_{y}^{\perp}\right\|=1+\left\|P_{x} P_{y}^{\perp}\right\|=1+\left\|P_{y}^{\perp} x\right\| .
$$

But on the other hand, as $\phi^{\prime}$ is an isometry, we know that $d_{\mathrm{S}}\left(P_{x}, P_{y}\right)$ $=d_{\mathrm{S}}\left(P_{x}, P_{y}^{\perp}\right)$. Hence $\left\|P_{y} x\right\|=\left\|P_{y}^{\perp} x\right\|$. Since, by Pythagoras' theorem, $\left\|P_{y} x\right\|^{2}+\left\|P_{y}^{\perp} x\right\|^{2}=1$, it follows that $|\langle x, y\rangle|=\left\|P_{y} x\right\|=1 / \sqrt{2}$, a contradiction. If $x$ and $y$ are arbitrary unit vectors, then we can choose a third unit vector $z$ such that both pairs $x, z$ and $z, y$ satisfy the above requirements. It now follows that $\phi^{\prime}$ is either the identity or taking the orthogonal complement on the set $\mathcal{P}_{1}(H)$ of all rank-one projections.

Composing $\phi^{\prime}$ if necessary with taking the orthogonal complement (which is a surjective isometry as we have learnt from the Proposition), there is no loss of generality in assuming that $\phi^{\prime}$ acts as the identity on $\mathcal{P}_{1}(H)$. It then remains to prove that $\phi^{\prime}$ is the identity on the set of all nontrivial projections. Pick a nontrivial $Q \in \mathcal{P}(H)$. We know that the rank-one projection $P$ does not commute with $Q$ if and only if $P=\phi^{\prime}(P)$ does not commute with the nontrivial projection $R=\phi^{\prime}(Q)$. Take a unit vector $x$ in $H$ which is neither in the range nor in the kernel of $Q$. Then the rank-one projection $P_{x}$ does not commute with $Q$. Therefore,

$$
d_{\mathrm{S}}\left(P_{x}, Q\right)=\left\|P_{x}+Q\right\|=1+\left\|P_{x} Q\right\|=1+\|Q x\|
$$

and similarly

$$
d_{\mathrm{S}}\left(P_{x}, R\right)=1+\|R x\| .
$$

For $d_{\mathrm{S}}\left(P_{x}, Q\right)=d_{\mathrm{S}}\left(\phi^{\prime}\left(P_{x}\right), \phi^{\prime}(Q)\right)=d_{\mathrm{S}}\left(P_{x}, R\right)$, it follows that

$$
\langle Q x, x\rangle=\|Q x\|^{2}=\|R x\|^{2}=\langle R x, x\rangle .
$$

Since this equality holds on a dense set of vectors $x$ in $H$, by continuity it holds for every $x \in H$. This shows that $Q=R=\phi^{\prime}(Q)$. Thus $\phi^{\prime}$ is the identity on the set of all nontrivial projections. Going back to the original transformation $\phi$ we see that either

$$
\phi(P)=U P U^{*} \quad(0, I \neq P \in \mathcal{P}(H)),
$$

or

$$
\phi(P)=U P^{\perp} U^{*} \quad(0, I \neq P \in \mathcal{P}(H)) .
$$

Observe that, as $\phi$ preserves commutativity in both directions, $\phi^{\prime}$ permutes the set of trivial projections. This completes the proof in the case when $\operatorname{dim} H \geq 3$. 
Finally, suppose that $\operatorname{dim} H=2$. In this case the rank-one projections are exactly the nontrivial projections. Restricting $\phi$ onto this set, we obtain a bijective map which preserves the so-called transition probability. Indeed, for any two different rank-one projections $P, Q$ one can easily verify that

$$
d_{\mathrm{S}}(P, Q)=\|P+Q\|=1+\|P Q\|=1+\sqrt{\operatorname{tr} P Q} .
$$

Therefore,

$$
\operatorname{tr} P Q=\operatorname{tr} \phi(P) \phi(Q) .
$$

Bijective maps on the set of rank-one projections with this property are called quantum mechanical symmetry transformations in quantum mechanics. According to the already mentioned theorem of Wigner, there exists a unitary or antiunitary operator $U$ on $H$ such that

$$
\phi(P)=U P U^{*}
$$

for every rank-one projection $P$ on $H$. Clearly, $\phi$ permutes the set of all trivial projections also in the present case, and hence the proof of the theorem is complete.

We conclude the paper with some remarks.

REMARKS. (1) Firstly, one may ask why taking the orthogonal complement does not appear in the proof above in the case $\operatorname{dim} H=2$. The answer is that it does appear in a certain way. In fact, one can readily check the following. If $e_{1}, e_{2}$ form an orthonormal basis in $H$, then defining

$$
U x=\left\langle e_{2}, x\right\rangle e_{1}-\left\langle e_{1}, x\right\rangle e_{2}
$$

for every $x \in H$, we obtain an antiunitary operator on $H$ such that

$$
U P U^{*}=P^{\perp}
$$

for every nontrivial projection $P$. Obviously, such an operator $U$ does not exist in higher dimensions.

(2) We have seen that the values of the metric $d_{\mathrm{S}}$ jump up from 0 to the interval $[1,2]$. It is natural to ask if they really run through the entire set $[1,2]$. Picking unit vectors $x, y \in H$ which are not constant multiples of each other, we see that

$$
d_{\mathrm{S}}\left(P_{x}, P_{y}\right)=\left\|P_{x}+P_{y}\right\|=1+\left\|P_{x} P_{y}\right\|=1+|\langle x, y\rangle| .
$$

This shows that $d_{\mathrm{S}}$ takes every value from the interval $[1,2[$. We assert that it also takes the value 2 if and only if the underlying space is infinitedimensional. Indeed, for the sufficiency, suppose without loss of generality that $H=l_{2}$. Consider the following closed subspaces in $H$ : let $M$ consist of all elements of $l_{2}$ of the form $\left(\xi_{1}, 0, \xi_{3}, 0, \xi_{5}, \ldots\right)$ and $N$ consist of all elements of $l_{2}$ of the form $\left(\eta_{1}, \eta_{1} / 2, \eta_{3}, \eta_{3} / 4, \eta_{5}, \eta_{5} / 6, \ldots\right)$. (We remark that $M$ and $N$ are standard examples showing that the sum of two closed subspaces may not be closed.) Then $M \cap N=\{0\}$ (and $M+N$ is a dense, proper subspace 
of $\left.l_{2}\right)$. It follows that $d_{\mathrm{S}}\left(P_{M}, P_{N}\right)=\left\|P_{M}+P_{N}\right\|$. Considering the vector $x_{k} \in l_{2}$ whose $(2 k-1)$ th coordinate is 1 , the next one is $1 /(2 k)$ and all the other coordinates are 0 , it follows that

$$
\frac{\left\|P_{M} x_{k}+P_{N} x_{k}\right\|^{2}}{\left\|x_{k}\right\|^{2}}=\frac{4+1 /\left(4 k^{2}\right)}{1+1 /\left(4 k^{2}\right)} .
$$

This sequence tends to 4 as $k$ goes to infinity. Hence, $d\left(P_{M}, P_{N}\right)=2$. For the necessity, suppose that $H$ is finite-dimensional. Assume that $P, Q \in \mathcal{P}(H)$ with $d_{\mathrm{S}}(P, Q)=2$. Clearly, $P, Q$ are not comparable. Applying the matrix representation introduced in the proof of the Proposition, we have

$$
2=d_{\mathrm{S}}(P, Q)=\left\|P_{0}+Q_{0}\right\|=1+\left\|P_{0} Q_{0}\right\| .
$$

So $\left\|P_{0} Q_{0}\right\|=1$. As we are in finite dimension, there is a unit vector $x$ such that $\left\|P_{0} Q_{0} x\right\|=1$, which implies that $\left\|Q_{0} x\right\|=1$. But this yields $Q_{0} x=x$ and so $\left\|P_{0} x\right\|=1$, which in the same manner implies that $P_{0} x=x$. Consequently, $x$ is in the ranges of both $P_{0}$ and $Q_{0}$, contrary to $P_{0} \wedge Q_{0}=0$. This shows that in finite dimension the values of $d_{\mathrm{S}}$ are all strictly less than 2 .

(3) In the proof of our theorem we have verified and then heavily used the fact that the surjective isometries are commutativity preserving transformations on the set of all projections. A deep analysis of commutativity preserving (and related) maps on the space of all idempotents has been carried out by P. Šemrl [10, 11].

Acknowledgements. The first author was supported by the Hungarian National Foundation for Scientific Research (OTKA) Grant No. NK68040 and by the Alexander von Humboldt Foundation, Germany.

\section{References}

[1] N. I. Akhiezer and I. M. Glazman, Theory of Linear Operators in Hilbert Space, Dover Publ., New York, 1993.

[2] P. Bjørstad and J. Mandel, On the spectra of sums of orthogonal projections with applications to parallel computing, BIT 31 (1991), 76-88.

[3] D. Buckholtz, Hilbert space idempotents and involutions, Proc. Amer. Math. Soc. 128 (1999), $1415-1418$.

[4] L. Molnár, Transformations on the set of all n-dimensional subspaces of a Hilbert space preserving principal angles, Comm. Math. Phys. 217 (2001), 409-421.

[5] -, Selected Preserver Problems on Algebraic Structures of Linear Operators and on Function Spaces, Lecture Notes in Math. 1895, Springer, 2006.

[6] -, Maps on the n-dimensional subspaces of a Hilbert space preserving principal angles, Proc. Amer. Math. Soc. 136 (2008), 3205-3209.

[7] L. Molnár and P. Šemrl, Non-linear commutativity preserving maps on self-adjoint operators, Quart. J. Math. 56 (2005), 589-595.

[8] J. Pykacz and E. Santos, Hidden variables in quantum logic approach reexamined, J. Math. Phys. 32 (1991), 1287-1292. 
[9] E. Santos, The Bell inequalities as tests of quantum logics, Phys. Lett. A 115 (1986), 363-365.

[10] P. Semrl, Maps on idempotents, Studia Math. 169 (2005), 21-44.

[11] —, Maps on idempotent matrices over division rings, J. Algebra 298 (2006), 142187.

[12] U. Uhlhorn, Representation of symmetry transformations in quantum mechanics, Ark. Fysik 23 (1963), 307-340.

[13] I. Vidav, The norm of the sum of two projections, Proc. Amer. Math. Soc. 65 (1977), $297-298$.

Institute of Mathematics

University of Debrecen

P.O. Box 12

H-4010 Debrecen, Hungary

E-mail: molnarl@math.klte.hu

http://www.math.klte.hu/ molnarl/
Institut für Analysis Technische Universität Dresden D-01062 Dresden, Germany E-mail: Werner.Timmermann@tu-dresden.de

Received August 27, 2008

Revised version October 13, 2008 